\title{
Strong Conformal Dynamics at the LHC and on the Lattice
}

\author{
Markus A. Luty \\ Physics Department, University of California Davis \\ Davis, California 95616
}

\begin{abstract}
Conformal technicolor is a paradigm for new physics at the LHC that may solve the problems of strong electroweak symmetry breaking for quark masses and precision electroweak data. We give explicit examples of conformal technicolor theories based on a QCD-like sector. We suggest a practical method to test the conformal dynamics of these theories on the lattice.
\end{abstract}




\section{Conformal Technicolor}

Electroweak symmetry breaking by strong dynamics at the $\mathrm{TeV}$ scale has great theoretical appeal, but has well-known difficulties with fermion masses and precision electroweak data. Conformal technicolor [1] is a framework closely related to "walking" technicolor [2] in which there are good reasons to think that these problems can be solved. Whether they are in fact solved depends on the dynamics of stronglycoupled small- $N$ conformal field theories, about which very little is known. In this paper we construct simple models of conformal technicolor based on QCD in the "conformal window." We also propose a practical method to measure the scaling dimension of the operator $\bar{\psi}_{L} \psi_{R}$. This gives a new lattice probe of conformal dynamics, and measures one of the most important parameters in conformal technicolor models. The reader interested only in the lattice aspects may wish to skip to section 3.

To motivate our discussion, we begin by reviewing the problems with strong electroweak symmetry breaking and how they are addressed in conformal technicolor. We begin with fermion masses. The most straightforward approach to generate fermion masses is to couple the standard model fermions directly to the operator $\Phi$ whose VEV breaks electroweak symmetry:

$$
\Delta \mathcal{L} \sim \frac{1}{M^{d-1}} \bar{Q}_{L} q_{R} \Phi
$$

where $d$ is the dimension of $\Phi$. We will be interested in the case where $\Phi$ is a technifermion bilinear of the form $\bar{\psi}_{L} \psi_{R}$. If the theory has QCD-like dynamics then $d=3$, but we will see that smaller values are allowed. The term Eq. (1.1) is therefore a higher-dimension interaction that requires new physics at a scale $M \sqrt[1]{\square}$

The flavor problems are of two kinds. First, there is the problem of whether the new physics at the scale $M$ also generates operators of the form

$$
\Delta \mathcal{L} \sim \frac{1}{M^{2}}\left(\bar{Q}_{L} q_{R}\right)^{2}
$$

These potentially give rise to flavor-changing neutral currents. This is a serious problem in "extended technicolor" models in which the operators Eq. (1.1) are generated by massive gauge bosons coupling the technifermions and standard model fermions [7].

\footnotetext{
${ }^{1}$ Alternatively, it is possible that standard model fermions get their mass by mixing with composite states [3, an idea that arises naturally in the context of Randall-Sundrum models [4] with fermions in the bulk [5. These models are "dual" descriptions of strongly coupled conformal theories [6. These have a somewhat different set of flavor problems (reviewed for strongly coupled small- $N_{c}$ theories in Ref. [1]), and we will focus on models based on Eq. (1.1).
} 
The second flavor problem is the fact that the operator Eq. (1.1) gets strong at a scale that is not far above the $\mathrm{TeV}$ scale. For QCD-like technicolor this scale is $\sim 3 \mathrm{TeV}$, while for a walking theory with $d=2$ it is $\sim 10 \mathrm{TeV}$. This means that in this framework the dynamics that generates the top quark mass cannot be separated from the dynamics that breaks electroweak symmetry. Models in which the interactions responsible for fermion masses are strong and play a role in electroweak symmetry breaking are discussed in Refs. [8]. The required strong dynamics is similar to those in the Nambu-Jona-Lasinio model [9], and requires fine-tuning and additional dynamical assumptions [10].

Strong electroweak symmetry breaking also has potential problems with precision electroweak constraints. Using only "naïve dimensional analysis" [11] to estimate the contribution from the strongly-coupled sector, the situation is illustrated in Fig. 1. From this alone we would conclude that fitting the data does not require any tuning. However, this requires that the strongly coupled theory give $S<0$. For QCD-like theories one finds quite robustly that $S>0$ [12]. Extended technicolor theories can have negative contributions to $S$ from pseudo-Nambu-Goldstone bosons [13]. Also, studies of "walking" technicolor suggest that the strong-coupling contribution to $S$ is significantly smaller than in QCD-like technicolor, and may even be negative [14]. The theories we discuss below have dynamics similar to walking, and therefore some of these mechanisms may reduce $S$ compared with the estimate from scaling up QCD. At minimum, we can make the conservative statement that the theories discussed here do not have QCD-like dynamics, and there is nothing that excludes the possibility that they have small $S$, or even $S<0$. We conclude that a good electroweak fit is possible due to non-QCD-like strong dynamics at the $\mathrm{TeV}$ scale and/or new light states below the TeV scale.

Of the three problems reviewed above, the tension with precision electroweak physics is generally viewed as the most problematic. It is indeed a striking fact that a weakly coupled extension of the standard model with a light Higgs boson (e.g. SUSY) naturally satisfies all electroweak constraints. However, the minimal supersymmetric standard model suffers from the fact that the Higgs is too light, requiring either fine-tuning or significant amount of non-minimal structure. An alternative is "little Higgs" models [15] and their cousins [16] that solve only the "little" hierarchy problem associated with precision electroweak data. These also require carefully constructed new sectors, and achieve only technical naturalness up to scales of order $10 \mathrm{TeV}$. Given these difficulties, it seems worthwhile to explore the alternative possibility that the agreement of the standard model with precision electroweak data is somewhat accidental. 


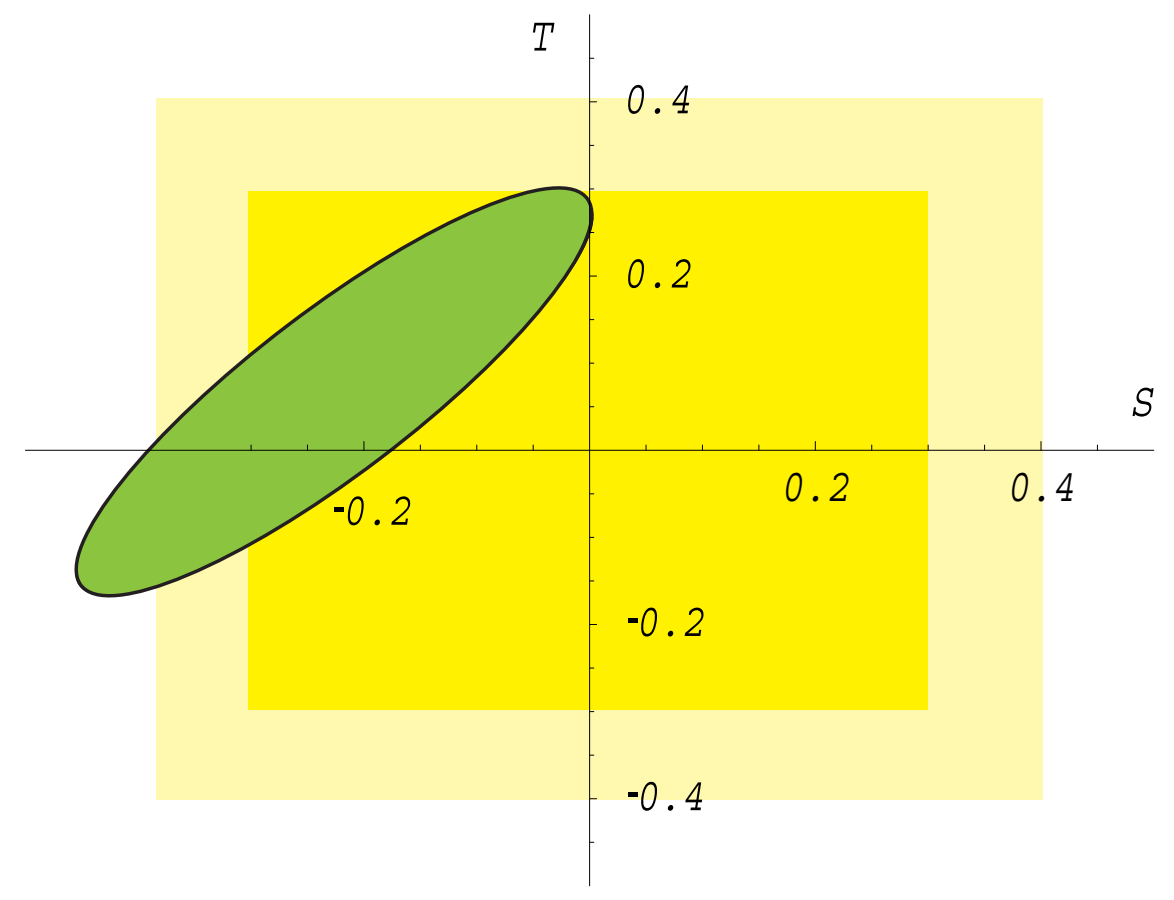

Fig. 1. 95\% confidence level constraints on $S$ and $T$ from precision electroweak data. The constraints are computed for a reference Higgs mass of $500 \mathrm{GeV}$, so the values correspond to the high-energy contribution in a strongly coupled theory. The shaded squares correspond to the estimates $S, T \sim \pm 1 / \pi$ for a strongly coupled theory.

The problem with flavor-changing neutral currents depends on UV physics, and is very model-dependent. One simple solution is that the operators Eq. (1.1) are generated by scalar Higgs exchange in a theory with SUSY broken at a scale $\gtrsim$ $10 \mathrm{TeV}$, an idea known as "bosonic technicolor" [17]. Since all 4-fermion operators are proportional to Yukawa couplings, the operators Eq. (1.2) are diagonal in the same basis as Eq. (1.1) (up to small renormalization group effects) and flavor-changing neutral currents are sufficiently small. It may appear implausible to have both SUSY and technicolor, but it is worth remembering that weak-scale SUSY also has a flavor problem from squark mass mixing. In bosonic technicolor, SUSY and technicolor each solve the flavor problem of the other. SUSY brings flavor structure down from the far UV, while strong dynamics is responsible for breaking electroweak symmetry while suppressing the flavor-violating effects of squark masses.

This leaves us with the top quark. We view this as the most serious problem, 
since it requires new physics near the $\mathrm{TeV}$ scale. This is the problem that motivated conformal technicolor. In this framework the electroweak symmetry breaking sector is near a strongly-coupled conformal fixed point above the $\mathrm{TeV}$ scale. In a general conformal field theory, the dimension of a scalar operator such as $\Phi$ is constrained only to be larger than 1 [18]. For example, if $d=1.5$ the top quark operator gets strong at a scale of order $30 \mathrm{TeV}$. If the flavor physics is a bosonic technicolor theory, we only require the top quark coupling to be weakly coupled at $10 \mathrm{TeV}$, in which case $d \lesssim 1.5$ is probably sufficient.

Can we really have such large anomalous dimensions? If $d<2$ then naïvely the singlet operator $\Phi^{\dagger} \Phi$ has dimension $2 d<4$. Then the theory has a relevant operator that cannot be forbidden by any symmetry from appearing in the Lagrangian, and the fixed point is not IR stable. The correct general formulation is that $\Phi^{\dagger} \Phi$ is the singlet operator of lowest dimension in the OPE of $\Phi$ and $\Phi^{\dagger}$. This operator need not have twice the dimension of $\Phi$ due to anomalous dimensions. In perturbation theory the anomalous dimensions are small, but they can be order 1 in a strongly coupled theory. We cannot get arbitrarily close to $d=1$, because in the limit $d \rightarrow 1 \Phi$ becomes a weakly coupled scalar field [18] and the dimension of $\Phi^{\dagger} \Phi$ approaches 2. We also cannot have $d<2$ in a large- $N_{c}$ theory, because in these theories the dimension of $\Phi^{\dagger} \Phi$ is equal to $2 d+\mathcal{O}\left(1 / N_{c}\right)$ even at strong coupling. The same phenomenon occurs in Randall-Sundrum models, which can be viewed as "dual" descriptions of large- $N_{c}$ conformal field theories [1].

Studies of walking technicolor using the gap equation [19] also find $d \geq 2$ [2, 20]. In the gap equation the condensate $\langle\bar{\psi} \psi\rangle$ forms precisely when the dimension of the operator $\bar{\psi} \psi$ approaches 2. The gap equation is an uncontrolled approximation that keeps only leading effects in the large- $N_{c}$ limit, and it is therefore not surprising that it reproduces the large- $N_{c}$ relation between the dimensions of $\bar{\psi} \psi$ and $(\bar{\psi} \psi)^{2}$.

The limit $d \rightarrow 1$ can be studied rigorously using general properties of conformal field theories. It was shown in Ref. [21] that the dimension of $\Phi^{\dagger} \Phi$ is $2 d+\mathcal{O}\left((d-1)^{1 / 2}\right)$ as $d \rightarrow 1$ [21]. This suggests that the anomalous dimension of $\Phi^{\dagger} \Phi$ may be large even if $d$ is small. Ref. [21] also gives a rigorous bound on the lowest dimension operator appearing the OPE of $\Phi^{\dagger} \Phi$, but the methods cannot distinguish the singlet from the non-singlet operators, so this does not give a bound on $d$.

We conclude that at present there is no known reason that an IR stable fixed point cannot have scalar operators with $d<2$. Such theories would significantly improve the prospects of overcoming the most serious problem with strong electroweak symmetry breaking, namely the top quark mass. 


\section{A Simple Model}

We argued above that a conformal field theory with $d<2$ must have both strong coupling and small $N_{c}$. A natural candidate theory is therefore $S U\left(N_{c}\right)$ gauge theory (with $N_{c}=2$ or 3 say) with $N_{f}$ flavors in the conformal window. It has been long known that $S U\left(N_{c}\right)$ QCD is conformal when the 1-loop beta function is sufficiently small, at least for large $N_{c}$ [22]. In supersymmetric QCD the work of Seiberg shows that conformal fixed points are much more generic: there is a large "conformal window" $\frac{3}{2}<N_{f} / N_{c}<3$ that exists even for small $N_{c}$. This is a strong hint that conformal fixed points may be a feature of non-supersymmetric QCD over a wide range of $N_{c}$ and $N_{f}$. Lattice studies have investigated this question and have found evidence for conformal fixed points. Iwasaki et. al. find evidence for an IR fixed point for $N_{c}=3$ and $7 \leq N_{f} \leq 16$ [23]. Appelquist et. al. find evidence for a fixed point at $N_{f}=12$ but not at $N_{f}=8$ [24], and Deuzeman et. al. also find that $N_{f}=8$ is in the confining phase [25]. The non-perturbative renormalization group study of Ref. 26] suggests that the fixed point exists for $12 \leq N_{f} \leq 16$. These results are encouraging for the existence of a strong conformal phase, although the discrepancies clearly need to be understood.

These can be compared with an extrapolation from the conformal window of SUSY QCD, which suggests a conformal window

$$
3.25 \lesssim \frac{N_{f}}{N_{c}} \lesssim 5.5 .
$$

for non-supersymmetric QCD [27]. This leads us to expect strongly-coupled fixed points for non-supersymmetric $S U(2)$ near $N_{f}=9$, and for $S U(3)$ near $N_{f}=13$.

We therefore consider an $S U\left(N_{c}\right)$ gauge theory with $N_{f}$ flavors assumed to be a strong conformal field theory. We weakly gauge this theory with the standard model so that 2 of the flavors form a minimal technicolor sector [28] while the remaining flavors are electroweak singlets. That is, under the gauge group $S U\left(N_{c}\right)_{\mathrm{CTC}} \times S U(2)_{\mathrm{W}} \times$ $U(1)_{\mathrm{Y}}$ the fields are

$$
\begin{aligned}
\psi_{L} & \sim\left(N_{c}, 2\right)_{0}, \\
\psi_{R} & \sim\left(N_{c}, 1\right)_{\frac{1}{2}}, \\
\psi_{R}^{\prime} & \sim\left(N_{c}, 1\right)_{-\frac{1}{2}},
\end{aligned}
$$

together with $N_{f}-2$ copies of

$$
\begin{aligned}
& \chi_{L} \sim\left(N_{c}, 1\right)_{0}, \\
& \chi_{R} \sim\left(N_{c}, 1\right)_{0} .
\end{aligned}
$$


We also assume that the theory has an explicit mass term for the electroweak singlet $\chi$ fields

$$
\Delta \mathcal{L}=m_{\chi} \bar{\chi}_{L} \chi_{R}+\text { h.c. }
$$

(For $N_{c}=2$ there is no invariant difference between $\chi_{L}$ and $\chi_{R}$ except as defined by this mass term.) Theories of this kind have been considered previously [29] with different dynamical assumptions (see section 4 below).

This theory is assumed to be at a conformal fixed point above the $\mathrm{TeV}$ scale. The explicit mass term is a relevant operator that forces the theory to flow away from the fixed point at the $\mathrm{TeV}$ scale. Below the $\mathrm{TeV}$ scale, we expect the theory to be in the same universality class as the theory with 2 massless flavors. That is, we expect that the theory confines and breaks chiral symmetry just like minimal technicolor. A heuristic picture is that below the scale of the $\chi$ mass term the gauge coupling is no longer prevented from blowing up by the $\chi$ fermions, and therefore confines just like the theory with 2 massless flavors. (This sort of straightforward decoupling is known to hold in supersymmetric QCD.) Of course, this is another dynamical assumption that should be checked.

Because the theory is in a nontrivial fixed point the operator $\bar{\chi}_{L} \chi_{R}$ will have a nontrivial scaling dimension $d \neq 3$ in general. The theory has an anomaly-free $U\left(N_{f}\right)$ flavor symmetry that means that this has the same dimension as the operators $\bar{\psi}_{L} \psi_{R}$ and $\bar{\psi}_{L} \psi_{R}^{\prime}$ that generate the top quark mass. We assume that $d \lesssim 1.5$ to allow the top quark to be weakly coupled to the strong sector.

This means that the $\chi$ mass term is a relevant operator. A dimensionless measure of the strength of the coupling $m_{\chi}$ at the scale $\mu$ is

$$
\hat{m}_{\chi}(\mu) \sim \frac{m_{\chi}\left(\mu_{0}\right)}{\mu_{0}}\left(\frac{\mu_{0}}{\mu}\right)^{4-d} .
$$

Here $\mu_{0}$ is a reference renormalization scale. The scale $\mu$ where $\hat{m}_{\chi} \sim 1$ determines the scale of conformal symmetry breaking:

$$
\Lambda_{\mathrm{CTC}}=\mu_{0}\left(\frac{m_{\chi}\left(\mu_{0}\right)}{\mu_{0}}\right)^{1 /(4-d)} .
$$

Since the coupling is already strong at the fixed point, we expect the transition to the new phase to be rapid. Since we assume that this new phase confines like 2flavor QCD, we identify $\Lambda_{\mathrm{CTC}} \sim \mathrm{TeV}$ with the scale of strong electroweak symmetry breaking. 
In this model, the scale of electroweak symmetry breaking is put in "by hand" in terms of the value of the $\chi$ mass term. The important point is that the smallness of the $\chi$ mass term is protected by a chiral symmetry, so this is a perfectly natural solution of the hierarchy problem. In fact, this is closely analogous to the mechanism that protects the hierarchy in supersymmetric models. There is an extension of spacetime symmetry (conformal symmetry) that forbids masses for fields in the symmetry breaking sector, and this symmetry is explicitly but softly broken to give an explanation for the smallness of the weak scale compared to e.g. the Planck scale.

\section{Conformal Dynamics on the Lattice}

The conformal technicolor models described above rely on the conformal dynamics of $S U\left(N_{c}\right)$ gauge theories with $N_{f}$ flavors. The most important of these are the existence of the conformal fixed point for suitable values of $N_{f}$ and $N_{c}$ and the dimension $d$ of the order parameter $\bar{\psi}_{L} \psi_{R}$. We now describe how these can be addressed on the lattice.

We consider the theory with nonzero fermion masses for all flavors. This completely breaks both chiral and conformal symmetry, allowing a practical test of the strong $S U\left(N_{c}\right)$ dynamics we we will see. To begin with, we take all masses to have a common value $m$. The scales in the lattice simulation must satisfy

$$
L^{-1} \ll m_{\text {had }} \ll \Lambda \ll a^{-1},
$$

where $L$ is the lattice size, $m_{\text {had }}$ is a low-lying hadron mass (see below), $\Lambda$ is the scale where the gauge coupling gets strong, and $a$ is the lattice spacing. The first two inequalities are required so that the physics at the hadronic mass scale is probed in the simulation; the final inequality is required to ensure that the gauge coupling at the lattice scale is weak, and therefore the lattice simulation is probing the desired continuum limit. Lattice simulations have a limited dynamical range, and these hierarchies will not be large in practice.

To distinguish the confining and conformal phases of gauge theory, we use the hadron masses. We first discuss the more familiar confining phase. Assuming that the quark masses are sufficiently small, the theory confines at the scale $\Lambda$, and spontaneously breaks the $S U\left(N_{f}\right) \times S U\left(N_{f}\right)$ symmetry down to $S U\left(N_{f}\right)$. This results in $N_{f}^{2}-1$ pseudo Nambu-Goldstone bosons. They will have mass related to the quark mass $m$ by

$$
m_{\pi}=c_{\pi}(\Lambda m)^{1 / 2}
$$


for small $m$. Other mesons such as the $\rho$ will have masses independent of $m$ at leading order:

$$
m_{\rho}=c_{\rho} \Lambda
$$

The constants $c_{\pi}$ and $c_{\rho}$ depend on the precise definition of $m$ and $\Lambda$, but the scaling with $m$ is unambiguous, since both $m$ and $\Lambda$ are well-defined up to a multiplicative constant.

In the conformal case, the theory enters a conformal phase at scales below $\Lambda$. The quark mass term breaks the conformal symmetry explicitly, and we expect that the theory below the conformal symmetry breaking scale has a nonzero mass gap. Note that the global symmetry of this model is a vectorlike $U\left(N_{f}\right)$ symmetry, which cannot be spontaneously broken [30]. Therefore, there can be no massless Nambu-Goldstone bosons in this theory. The absence of a chiral symmetry also means that we do not expect massless composite fermions in this theory $\mathrm{L}^{2}$

If conformal symmetry is broken at scales smaller than $\Lambda$, the conformal symmetry breaking scale is effectively the only scale in the theory. All hadron masses in this theory are therefore proportional to this scale, which is given by Eq. (2.6):

$$
\text { conformal: } m_{\pi, \rho}=\tilde{c}_{\pi, \rho} \Lambda\left(\frac{m}{\Lambda}\right)^{1 /(4-d)} \text {. }
$$

As in the confining phase, the scaling with $m$ holds independently of the precise definition of $m$ and $\Lambda$.

We see that measurement of hadron masses in the theory with quark masses gives a direct test of the conformal dynamics. What is important is the scaling of the hadron masses with the quark masses, with the hadronic scale $\Lambda$ held fixed. As long as the quark masses are sufficiently small, this is equivalent to holding the bare (lattice) coupling fixed. The scaling with the quark masses is then well-defined as long as the quark masses are only multiplicatively renormalized.

One way to measure the hadron masses is to use 2-point functions $\left\langle\mathcal{O}^{\dagger}(0) \mathcal{O}(x)\right\rangle$, where $\mathcal{O}$ is an operator with the quantum numbers of the hadron of interest. As long as the operator $\mathcal{O}$ does not have vacuum quantum numbers, the 2 -point function is dominated by the lightest particle $\varphi$ with the quantum numbers of $\mathcal{O}$ for $|x| \gg 1 / m_{\varphi}$. For $|x| \ll L$ this is a simple exponential dependence $\langle\mathcal{O}(0) \mathcal{O}(x)\rangle \sim e^{-m_{\varphi}|x|}$. For $|x| \sim L$ the dependence on $m_{\varphi}$ is more complicated, but is completely determined by the geometry.

\footnotetext{
${ }^{2}$ It is possible that the theory flows to a different conformal fixed point, in which case there is no mass gap. This will show up as a power law volume dependence of correlation functions (see below).
} 
Another possibility is to use finite-volume scaling to measure the lightest hadron mass. This has been used extensively in studies of conformal systems in statistical mechanics [31] and in lattice gauge theory [32]. Here we focus on expectation values of gauge-invariant local singlet operators $\mathcal{O}$ such as $\bar{\psi} \psi$ or $\operatorname{tr} F^{\mu \nu} F_{\mu \nu}$. For finite $L$, the leading volume dependent effects come from the lightest states propagating from one side of the lattice to the other. (We assume that the lattice has periodic boundary conditions, so there is a discrete translation symmetry and therefore no preferred position on the lattice.) In the confining case the matrix element $\langle\pi|\mathcal{O}| \pi\rangle$ is nonzero, so the leading finite volume effects are controlled by the single pion states. In the conformal case, the mass of the lightest hadron is set by Eq. (3.4). In both cases, calling the lightest hadron $\varphi$, we have for $L \gg m_{\varphi}^{-1}$

$$
\langle\mathcal{O}\rangle=C_{0}+C_{1} e^{-m_{\varphi} L}+\text { independent of } L
$$

Different operators will give different values of $C_{0}$ and $C_{1}$, but they have the same exponential falloff with $m_{\varphi}$, so using different operators can be used as a consistency check. A disadvantage of this way of measuring the mass gap is that the exponential dependence must be extracted from on top of a larger constant. Furthermore, all particles contribute to the volume dependence, so there may be several particles with similar masses contributing to the exponential.

Measuring the dependence of the hadron masses on the quark mass is also useful to check the other main dynamical assumption of conformal technicolor theories, namely the spontaneous breaking of chiral symmetry in the theory with 2 massless flavors. To probe this, we give 2 of the flavors a smaller quark mass $m^{\prime} \ll m$. If the theory behaves as we have assumed above, we have

$$
\begin{aligned}
& m_{\pi} \propto\left[m^{\prime} m^{1 /(4-d)}\right]^{1 / 2} \\
& m_{\rho} \propto m^{1 /(4-d)}
\end{aligned}
$$

Here $\pi$ is a pseudo-Nambu-Goldstone boson associated with the breaking of the $S U(2) \times S U(2)$ chiral symmetry of the light flavors, while $\rho$ is any other hadron. Measuring this requires a further hierarchy of lattice scales, and will therefore be more costly to simulate.

\section{Conformal Versus "Walking" Dynamics}

We have not discussed the possibility of "walking" dynamics because we feel that it is less likely to occur than the conformal dynamics we are discussing. Recall that 
"walking" is the assumption that the gauge coupling is large over a large range of scales, but eventually blows up in the IR, leading to confinement and chiral symmetry breaking.

Conformal field theory gives a well-defined physical framework that can be used to address the question of walking dynamics. This has already been noted by many authors in the literature on walking technicolor [2, 20]. The assumption of walking is equivalent to the assumption that the theory is near a conformal fixed point for a range of energies, but does not flow to the fixed point in the deep IR. This means that the fixed point is unstable in the IR, i.e. there is a relevant operator in the Wilsonian effective action at the scale $\Lambda$ where the theory first becomes strongly coupled. The fact that the walking theory nonetheless comes close to the fixed point means that the relevant operator has a small coefficient in the effective Lagrangian, and/or the dimension of the relevant operator is close to 4 . Either scenario requires the presence of a small parameter.

In a theory with a large number of colors $N_{c}$ and flavors $N_{f}$, the anomalous dimensions of operators are a function of $N_{f} / N_{c}$, which becomes effectively a continuous variable. It is therefore plausible that in such theories there is a singlet operator whose dimension approaches 4 at a critical value of $N_{f} / N_{c}$. Such a theory could indeed exhibit walking behavior. Even then, one must assume that the relevant operator has a suffuciently small coefficient in the Wilsonian effective action at the scale $\Lambda$ to come close to the fixed point. However, a large- $N_{c}$ walking technicolor theory gives values of $S$ and $T$ enhanced by a factor of $N_{c}$, and is therefore not a good candidate for a theory of electroweak symmetry breaking. A viable (small $N_{c}$ ) walking theory requires an additional unexplained small parameter in order to come close to an IR unstable conformal fixed point.

It is for this reason that we believe that conformal dynamics is more robust than walking dynamics. Ultimately, however it is experiment that must decide. A practical method of looking for walking dynamics is the Schrödinger functional technique 33] used by Ref. [24]. This computes a non-perturbative running gauge coupling where the scale parameter is the volume of the system, and can be evaluated over a large range of scales. It is worth noting that the results of Ref. [24] obtained with this method for $N_{c}=3, N_{f}=12$ shows no sign of exiting the fixed point, and therefore indicate conformal rather than walking dynamics. 


\section{Conclusions}

We have argued that strong conformal dynamics can give an elegant solution of the hierarchy problem, while addressing the problems of strong electroweak symmetry breaking. Whether these ideas have anything to do with reality will be decided by experiments, both at the LHC and on the lattice. Investigating strong conformal dynamics on the lattice is also an interesting problem in its own right. We have suggested a simple method using the scaling dependence of hadron masses on the quark mass to find evidence for conformal dynamics and measure the scaling dimension of the operator $\bar{\psi} \psi$. We hope that these methods will prove useful and lead to further investigation in this area.

\section{Acknowledgements}

I would like to thank the organizers of the "Lattice Gauge Theory for LHC Workshop" at Lawrence Livermore Laboratory May 2008, for an invitation to speak that stimulated this work. I thank N. Christ and K. Holland for helpful comments on lattice simulations. I thank S. Chang and T. Okui for comments on the manuscript.

\section{References}

[1] M. A. Luty and T. Okui, JHEP 0609, 070 (2006) arXiv:hep-ph/0409274.

[2] B. Holdom, Phys. Rev. D 24, 1441 (1981); Phys. Lett. B 150, 301 (1985); K. Yamawaki, M. Bando and K. Matumoto, Phys. Rev. Lett. 56, 1335 (1986); M. Bando, K. Matumoto and K. Yamawaki, Phys. Lett. B 178, 308 (1986); T. W. Appelquist, D. Karabali and L. C. R. Wijewardhana, Phys. Rev. Lett. 57, 957 (1986); T. Appelquist and L. C. R. Wijewardhana, Phys. Rev. D 35, 774 (1987); Phys. Rev. D 36, 568 (1987).

[3] D. B. Kaplan, Nucl. Phys. B 365, 259 (1991).

[4] L. Randall and R. Sundrum, Phys. Rev. Lett. 83, 3370 (1999) arXiv:hepph/9905221.

[5] S. J. Huber and Q. Shafi, Phys. Lett. B 498, 256 (2001) arXiv:hep-ph/0010195;

K. Agashe, A. Delgado, M. J. May and R. Sundrum, JHEP 0308 (2003) 050 arXiv:hep-ph/0308036]; K. Agashe, R. Contino and R. Sundrum, Phys. Rev. Lett. 95, 171804 (2005) arXiv:hep-ph/0502222. 
[6] N. Arkani-Hamed, M. Porrati and L. Randall, JHEP 0108, 017 (2001) arXiv:hepth/0012148; R. Rattazzi and A. Zaffaroni, JHEP 0104, 021 (2001) arXiv:hepth/0012248].

[7] S. Dimopoulos and L. Susskind, Nucl. Phys. B 155, 237 (1979); E. Eichten and K. D. Lane, Phys. Lett. B 90, 125 (1980).

[8] T. Appelquist, M. Einhorn, T. Takeuchi and L. C. R. Wijewardhana, Phys. Lett. B 220, 223 (1989); R. S. Chivukula, A. G. Cohen and K. D. Lane, Nucl. Phys. B 343, 554 (1990); C. T. Hill, Phys. Lett. B 266, 419 (1991); Phys. Lett. B 345, 483 (1995) arXiv:hep-ph/9411426.

[9] Y. Nambu and G. Jona-Lasinio, Phys. Rev. 122, 345 (1961); Phys. Rev. 124, 246 (1961).

[10] M. A. Luty, Phys. Rev. D 48, 1295 (1993).

[11] A. Manohar and H. Georgi, Nucl. Phys. B 234, 189 (1984); H. Georgi, Weak Interactions And Modern Particle Theory, Benjamin/Cummings (Menlo Park, 1984).

[12] M. Golden and L. Randall, Nucl. Phys. B 361, 3 (1991); B. Holdom and J. Terning, Phys. Lett. B 247, 88 (1990); M. E. Peskin and T. Takeuchi, Phys. Rev. Lett. 65, 964 (1990); Phys. Rev. D 46, 381 (1992).

[13] M. A. Luty and R. Sundrum, Phys. Rev. Lett. 70, 529 (1993) arXiv:hepph/9209255].

[14] T. Appelquist and G. Triantaphyllou, Phys. Lett. B 278, 345 (1992); R. Sundrum and S. D. H. Hsu, Nucl. Phys. B 391, 127 (1993) |arXiv:hep-ph/9206225|; T. Appelquist and F. Sannino, Phys. Rev. D 59, 067702 (1999) arXiv:hep-ph/9806409.

[15] N. Arkani-Hamed, A. G. Cohen, E. Katz, A. E. Nelson, T. Gregoire and J. G. Wacker, JHEP 0208, 021 (2002) arXiv:hep-ph/0206020]; N. Arkani-Hamed, A. G. Cohen, E. Katz, A. E. Nelson, T. Gregoire and J. G. Wacker, JHEP 0208, 021 (2002) arXiv:hep-ph/0206020; D. E. Kaplan and M. Schmaltz, JHEP 0310, 039 (2003) arXiv:hep-ph/0302049.

[16] Z. Chacko, H. S. Goh and R. Harnik, Phys. Rev. Lett. 96, 231802 (2006) arXiv:hep-ph/0506256]; G. Burdman, Z. Chacko, H. S. Goh and R. Harnik, JHEP 0702, 009 (2007) arXiv:hep-ph/0609152.

[17] S. Samuel, Nucl. Phys. B 347, 625 (1990); M. Dine, A. Kagan and S. Samuel, Phys. Lett. B 243, 250 (1990). 
[18] G. Mack, Commun. Math. Phys. 55, 1 (1977). A simplified derivation is given in B. Grinstein, K. A. Intriligator and I. Z. Rothstein, Phys. Lett. B 662, 367 (2008) arXiv:0801.1140 [hep-ph]].

[19] K. Johnson, M. Baker and R. Willey, Phys. Rev. 136, B1111 (1964).

[20] A. G. Cohen and H. Georgi, Nucl. Phys. B 314, 7 (1989); T. Appelquist, J. Terning and L. C. R. Wijewardhana, Phys. Rev. Lett. 79, 2767 (1997) arXiv:hep$\mathrm{ph} / 9706238$.

[21] R. Rattazzi, V. S. Rychkov, E. Tonni and A. Vichi, JHEP 0812, 031 (2008) arXiv:0807.0004 [hep-th]].

[22] T. Banks and A. Zaks, Nucl. Phys. B 196, 189 (1982).

[23] Y. Iwasaki, K. Kanaya, S. Kaya, S. Sakai and T. Yoshie, Phys. Rev. D 69, 014507 (2004) arXiv:hep-lat/0309159.

[24] T. Appelquist, G. T. Fleming and E. T. Neil, Phys. Rev. Lett. 100, 171607 (2008) [arXiv:0712.0609 [hep-ph]]; arXiv:0901.3766 [hep-ph].

[25] A. Deuzeman, M. P. Lombardo and E. Pallante, arXiv:0804.2905 [hep-lat].

[26] J. Braun and H. Gies, Phys. Lett. B 645, 53 (2007) arXiv:hep-ph/0512085.

[27] T. Appelquist, A. Ratnaweera, J. Terning and L. C. R. Wijewardhana, Phys. Rev. D 58, 105017 (1998) arXiv:hep-ph/9806472.

[28] S. Weinberg, Phys. Rev. D 13, 974 (1976); L. Susskind, Phys. Rev. D 20, 2619 (1979); S. Weinberg, Phys. Rev. D 19, 1277 (1979).

[29] D. D. Dietrich and F. Sannino, Phys. Rev. D 75, 085018 (2007) arXiv:hepph/0611341.

[30] C. Vafa and E. Witten, Nucl. Phys. B 234, 173 (1984).

[31] For a review, see e.g. J. L. Cardy, J. Phys. A17 L385 (1984).

[32] See e.g. M. Lüscher, Commun. Math. Phys. 104, 177 (1986); Commun. Math. Phys. 105, 153 (1986).

[33] M. Lüscher, R. Narayanan, P. Weisz and U. Wolff, Nucl. Phys. B 384, 168 (1992) arXiv:hep-lat/9207009]. 